\title{
Analysis of Dose Distribution According to the Initial Electron Beam of the Linear Accelerator: A Monte Carlo Study
}

\author{
Hyojun Park', Hyun Joon Choi', Jung-In Kim², Chul Hee Min ${ }^{1, *}$ \\ ${ }^{1}$ Department of Radiation Convergence Engineering, Yonsei University, Wonju, Korea; ${ }^{2}$ Department of Radiation Oncology, Seoul National University \\ Hospital, Seoul, Korea
}

\section{Original Research}

Received February 14, 2018

Revision March 19, 2018

Accepted March 22, 2018

Corresponding author: Chul Hee Min

Department of Radiation Convergence Engineering, Yonsei University,

1 Yonsedae-gil, Heungop-myeon,

Wonju 26493, Korea

Tel: +82-33-760-2891

Fax: +82-33-760-2891

E-mail:chmin@yonsei.ac.kr

This is an Open-Access article distributed under the terms of the Creative Commons Attribution NonCommercial License (http://creativecommons.org/ licenses/by-nc/4.0) which permits unrestricted noncommercial use, distribution, and reproduction in any medium, provided the original work is properly cited.

Copyright $\odot$ 2018 The Korean Association for Radiation Protection

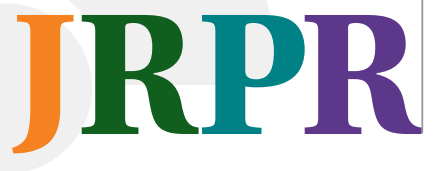

Background: Monte Carlo (MC) simulation is the most accurate for calculating radiation dose distribution and determining patient dose. In MC simulations of the therapeutic accelerator, the characteristics of the initial electron must be precisely determined in order to achieve accurate simulations. However, It has been computation-, labor-, and time-intensive to predict the beam characteristics through predominantly empirical approach. The aim of this study was to analyze the relationships between electron beam parameters and dose distribution, with the goal of simplifying the MC commissioning process.

Materials and Methods: The Varian Clinac 2300 IX machine was modeled with the Geant4 MC-toolkit. The percent depth dose (PDD) and lateral beam profiles were assessed according to initial electron beam parameters of mean energy, radial intensity distribution, and energy distribution.

Results and Discussion: The PDD values increased on average by $4.36 \%$ when the mean energy increased from $5.6 \mathrm{MeV}$ to $6.4 \mathrm{MeV}$. The PDD was also increased by $2.77 \%$ when the energy spread increased from $0 \mathrm{MeV}$ to $1.019 \mathrm{MeV}$. In the lateral dose profile, increasing the beam radial width from $0 \mathrm{~mm}$ to $4 \mathrm{~mm}$ at the full width at half maximum resulted in a dose decrease of $8.42 \%$ on the average. The profile also decreased by $4.81 \%$ when the mean energy was increased from $5.6 \mathrm{MeV}$ to $6.4 \mathrm{MeV}$. Of all tested parameters, electron mean energy had the greatest influence on dose distribution. The PDD and profile were calculated using parameters optimized and compared with the golden beam data. The maximum dose difference was assessed as less than $2 \%$.

Conclusion: The relationship between the initial electron and treatment beam quality investigated in this study can be used in Monte Carlo commissioning of medical linear accelerator model.

Keywords: Linear accelerator, Initial electron, Beam commissioning, Monte Carlo, Geant4

\section{Introduction}

Modern radiation therapy techniques that use a linear accelerator (LINAC), such as intensity modulated radiation therapy (IMRT), enable the dose distribution to better conform to the target volume with a small subfield sequence. To predict the patient dose, computerized treatment planning systems (TPS) have been used in the clinic. Most TPS use the pencil beam (PB) convolution algorithm, which obtains patient dose 
distributions using dose kernels consisting of previously calculated dose maps. This algorithm accelerates the dose calculation. However, the uncertainty of PB-based dose calculation has been reported to be high in heterogeneous tissue and in small-field beams due to the limited lateral scatter calculation [1-3]. For special cases requiring very accurate dose assessment, the TPS-based dose distribution must be verified with an independent dose evaluation method [1-3].

The Monte Carlo (MC) simulation is based on the premise that modeling is highly accurate. Thus, it has been regarded as the most accurate dose calculation method for radiation beams delivered into patients because it calculates the dose by considering each transported particle. This approach enables highly accurate dose calculation using a process similar to that of the actual beam delivery [2-5]. In this regard, the MC method is an important verification tool in the clinic. ${ }^{1)}$ However, its high computational burden has hindered its adoption, and the MC method is not currently available in the clinic [3, 6-8].

MC simulations for dose calculation begin by producing the accelerated electrons delivered to the target and the x-ray beam generated through the bremsstrahlung process. However, it is very difficult to determine the characteristics of the electron beam of interest. For example, (1) manufacturers do not provide standardized information about the initial electron beam, (2) the electron beam in the vacuum head cannot be directly measured, and (3) electron beams differ even when produced by the same model and also differ because of the aging effect [9-11]. To address these issues, indirect beam commissioning has been employed. This approach modifies various parameters of the initial electron beam to match the simulated MC beam quality with the measured quality by comparing the percent depth dose (PDD) and the lateral beam profile [9].

In previous studies for LINAC beam commissioning, initial beam spot size and mean electron energy [12] were chosen empirically by matching the simulated beam to the measurement by changing the electron characteristics $[13,14]$. While this simple method is the most commonly used method in beam commissioning [12-14], the results and conclusions reached with this method depend significantly on the researcher. To accurately and efficiently determine the characteristics of the electron beam, it is important to understand the relationship between the initial electron and the follow- ing Bremsstrahlung photon. This relationship is affected by the treatment head and is used as a decision-making standard during beam commissioning.

Tzedakis et al.[9] previously investigated the initial electron effect on the $6 \mathrm{MV}$ treatment beam. Tzedakis and colleagues based their work on the study by Bjork on the LINAC initial electron characteristics $[9,10]$ and used the Electron Gamma Shower 4 (EGS4) code for the Philips SL LINAC model. However, for 4D simulation with IMRT and image-guided radiation therapy (IGRT), Geometry And Tracking 4 (Geant4) is the preferred tool because Geant4 provides a flexible simulation setup including geometries and DICOM import for patient dose calculation, in addition to providing the same information as the TPS $[15,16]$. Moreover, precise modeling of the multileaf collimator (MLC), which determines the fluence map, is difficult without Geant 4 because of the complex geometry of this instrument.

The purpose of this study was to simplify the beam commissioning procedure and develop an accurate and efficient process for determining the optimal parameters for MC commissioning. To this end, the dose distributions, PDD values, and lateral profiles of the Varian Clinac 2300 IX machine were evaluated according to the characteristics of the initial electron beam using the Geant 4 toolkit.

\section{Materials and Methods}

\section{LINAC head modeling based on the Geant4 toolkit}

The Geant4 toolkit (ver. 4.9.6.p02), developed with the C++ language, was employed to simulate a $6 \mathrm{MV}$ photon beam produced by a Varian Clinac 2300 IX machine. The major components of the LINAC head, including the target, primary collimator, Beryllium window, flattening filter, ion chamber, mirror, jaws, and MLC, were modeled as shown in Figure 1. To achieve detailed modeling of the treatment head, blueprint supported by the machine vender was used as the reference when modeling the geometry.

\section{Evaluation of the dosimetric effect according to initial electron beam parameters}

To investigate the influence of (a) mean energy, (b) radial intensity distribution, and (c) energy spread on curve attributes such as depth of the maximum dose $\left(\mathrm{d}_{\max }\right)$ on the PDD and profile dose flatness, the PDDs and lateral dose profiles

1) Failla GA, Wareing T, Archambault Y, Thompson S. Acuros XB advanced dose calculation for the Eclipse treatment planning system. Palo Alto. CA: Varian Medical Systems. 2010;10-15. 


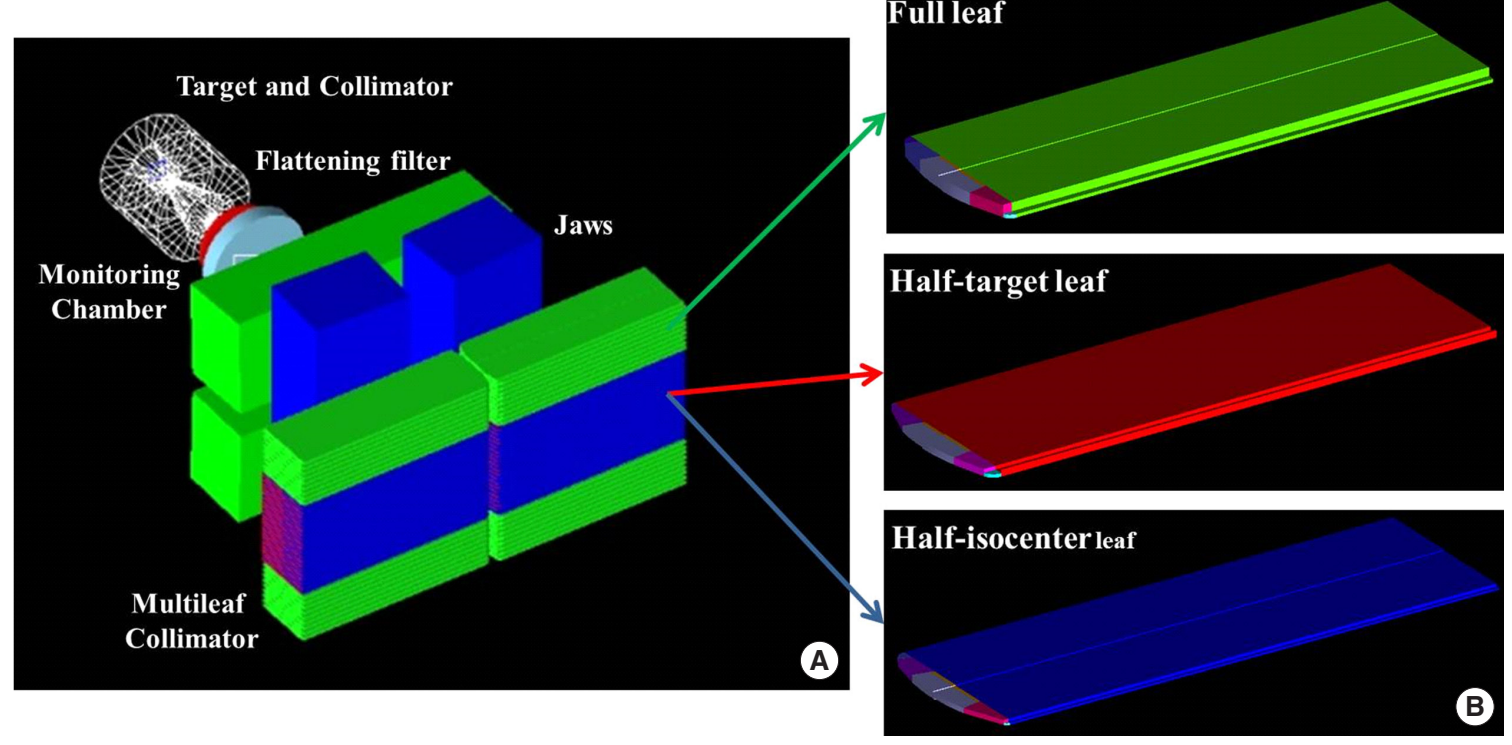

Fig. 1. Modeled LINAC head components (A) and MLC leaves (B) based on the Geant4 toolkit.

were calculated and analyzed with different initial electron beam conditions.

\section{1) Mean energy of the initial electron pencil beam}

To investigate the effect of mean energy on the PDD and the lateral beam profile, simulations were carried out while varying the mean initial electron beam energy from $5.6 \mathrm{MeV}$ to $6.4 \mathrm{MeV}$ in increments of $0.2 \mathrm{MeV}$. To evaluate only the influence of the mean electron energy on the dose distribution, the pencil beam was used, and the standard deviation of the energy was set to zero.

\section{2) Radial intensity distribution of the initial electron beam}

In a LINAC, the initial electron beam has a radial intensity distribution and an angular distribution because of the bending magnet, steering coils, and focusing coils in the electron transport system. The electron distribution was assumed to have a two-dimensional (2-D) Gaussian distribution, as suggested by the Keall et al. study. In this study, the distributions of the initial electrons generating the bremsstrahlung photon in the target were defined [17]. Karzmark et al. recommended that the electron angle should range from 0.06 to 0.3 degrees for the initial electron beam transport, which is close to zero. ${ }^{2)}$ Therefore, we assumed that the beam was parallel to the beam axis and only varied the full width at half maximum (FWHM) of the radial distribution (1 mm increments from $0 \mathrm{~mm}$ to $4 \mathrm{~mm}$ ). To assess the effect of electron beam radial width, the mean electron energy and energy spread were fixed at $6 \mathrm{MeV}$ and $0 \mathrm{MeV}$, respectively.

3) Energy spread of the initial electron pencil beam

The energy spread of the initial electron can be estimated with different waveguide types, and energy slits in the bending magnet orbit $[9,18]$. The electron spread from the accelerating waveguide has been reported to assume a Gaussian distribution according to Tanabe and Hamm's measurement [18].

We modeled the energy distribution of the initial electron as a Gaussian distribution and varied the FWHM from 0 $\mathrm{MeV}$ to $1.019 \mathrm{MeV}$ in $0.127 \mathrm{MeV}$ increments. A pencil beam was used, and the mean electron energy was set to $6 \mathrm{MeV}$.

4) Normalization and comparison of PDDs and profiles

To evaluate the relationships between the initial electron characteristics and the LINAC photon beam dose distributions, the PDDs and profiles of the various initial electron conditions were compared with those of the reference initial electron beam. The reference conditions of the initial electron beam were set to a mean energy of $6 \mathrm{MeV}$, a radial intensity of $0 \mathrm{~mm}$ at the FWHM, and an energy spread of $0 \mathrm{MeV}$.

The simulated PDDs and profiles were normalized to the dose values at $\mathrm{d}_{\max }$ and the distribution center, respectively.

2) Karzmark, C. J., Craig S. Nunan, and Eiji Tanabe. Medical electron accelerators. 1st edition. New York. McGraw-Hill. 1993. 
The PDD was assessed at $2 \mathrm{~mm}$ intervals at depths ranging from 0 to $50 \mathrm{~mm}$ to observe the dose distribution in build-up region and dose distribution nearby $\mathrm{d}_{\max }$. For depths greater than $50 \mathrm{~mm}$, the depth dose was assessed at $15 \mathrm{~mm}$ intervals for depths greater than $50 \mathrm{~mm}$ to reduce the statistical fluctuation. The depth before $d_{\max }$ was not considered in the PDD comparison for treatment beam modeling because electron equilibrium was not reached, and statistically significant fluctuation occurs in that region. Moreover, electron contamination affects the dose distribution before $\mathrm{d}_{\max }$.

In evaluating the profile, the simulated dose values were compared to those of the reference electron beam only in the in-field region in which the dose values showed more than $80 \%$ of the dose at the center, due to relatively high dose difference from few distance that can be regarded as ignorable in the dose fall-off region and in the out-field region. Also, high statistical error caused in the simulation because of the beam collimators such as jaws and the MLC. The relative dose difference was calculated to evaluate the PDD and lateral profile values. The relative dose difference was determined as follows:

Relative dose difference $(\%)=\frac{\left(\mathrm{D}_{\text {Calculated }}-\mathrm{D}_{\text {Reference }}\right) \times 100}{\mathrm{D}_{\text {Reference }}}$

\section{Simulation conditions for dose calculation}

In the simulation, G\$EmStandardPhysics_option3 was used because it is suitable for calculating transportations of photon and electron in medical applications $[19,20]$. To reduce the calculation time, variance reduction techniques were applied to components of the LINAC head, e.g., bremsstrahlung splitting in the target and Russian roulette in the downstream collimators. In addition, the phase space files containing information on the particles reaching the MLC (position, direction, kinetic energy, and particle type) were used to reduce the simulation time. The photon beam produced by more than $2.8 \times 10^{9}$ initial electrons was recorded in the phase space files and then used repeatedly four times to obtain the dose distribution with reduced statistical fluctuation.

In the simulation, the source-to-surface distance (SSD) was set to $100 \mathrm{~cm}$. The beam was delivered into a box-shaped voxelized water phantom measuring $50 \times 50 \times 40 \mathrm{~cm}^{3}$. Each voxel measured $5 \times 5 \times 2 \mathrm{~mm}^{3}$.

The PDD was calculated with a field size of $10 \times 10 \mathrm{~cm}^{2}$, which is generally considered to be the reference size for assessing the effects of initial electron parameters, while the profile was calculated at $d_{\max }$ with a field size of $40 \times 40 \mathrm{~cm}^{2}$. Since the profile with the larger beam field is more heavily impacted by changes to the initial electron conditions such as mean electron energy, energy spread, and beam radial width [10], the largest field size was selected for the profile assessment. Finally, the optimal parameters were determined by comparing the PDDs and profiles of golden beam data (GBD) achieved with beams of $4 \times 4 \mathrm{~cm}^{2}, 10 \times 10 \mathrm{~cm}^{2}$, and $30 \times 30 \mathrm{~cm}^{2}$ fields.

\section{Results}

\section{Effects of the initial electron beam on the PDD and profile}

1) Mean energy of the initial electron pencil beam

Figure 2 shows the PDDs and lateral dose profiles according to the initial electron energy. It also displays their differences compared to the reference initial electron beam. The electron beam with a mean energy of $5.8 \mathrm{MeV}$ showed a minimum PDD difference of about $0.24 \%$ from the reference electron beam, which had a mean energy of $6 \mathrm{MeV}$. Increasing the initial energy of the electron beam also increased the PDD values after $d_{\max }$. Specifically, the dose values increased on average by $4.36 \%$, while the mean energy increased from 5.6 to $6.4 \mathrm{MeV}$. This finding indicates that the overall PDD curve moved toward the beam direction with the higher electron energy, even though the reference parameters of the initial electron beam were the same.

In the lateral dose profile, the relative dose differences from the reference condition were decreased on average by $4.81 \%$ as the electron beam energy increased from 5.6 MeV to $6.4 \mathrm{MeV}$. The maximum dose difference in the horn with the reference energy of the electron beam, $6 \mathrm{MeV}$, was approximately $9.21 \%$ compared to that with the $6.4 \mathrm{MeV}$ electron beam. Under the initial electron reference conditions, the minimum difference in average dose between the two profiles was $0.56 \%$ and was found when the mean electron energy was $5.8 \mathrm{MeV}$ (Figure 2). Figure 2 indicates that the average initial electron energy is more sensitive to the lateral dose profile then the PDD, because dose difference change according to the mean electron energy increased more with the profile.

2) Radial intensity distribution of the initial electron beam

The PDD and lateral dose profile were calculated according to various electron beam radial intensity distributions. 

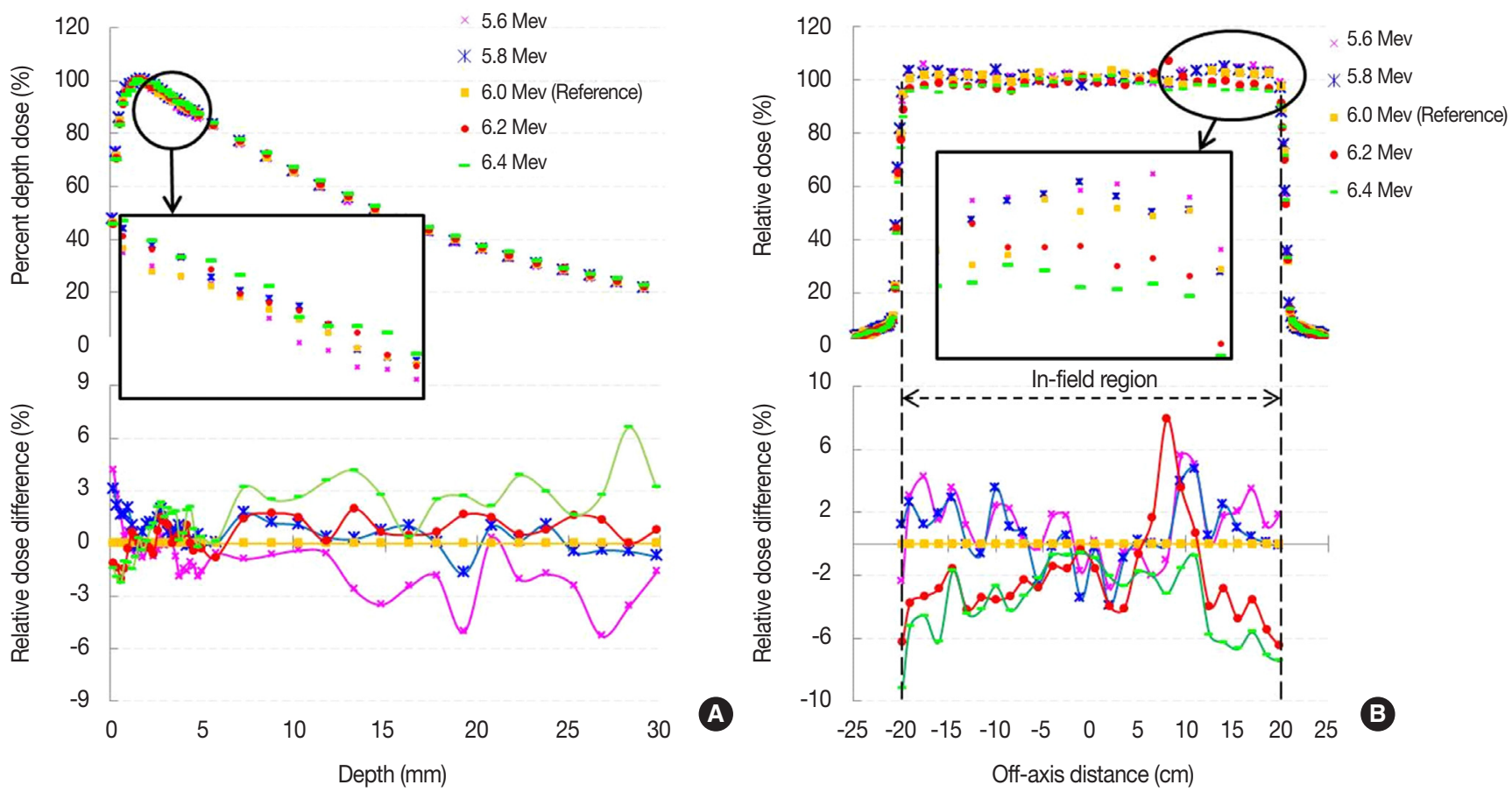

Fig. 2. Relative dose differences of percent depth dose (A) and lateral dose profile (B) from the reference initial electron beam according to average initial electron beam energy.

Differences from those of the reference electron beam are shown in Figure 3. For the PDD, the electron beam with a radial width of $1 \mathrm{~mm}$ at the FWHM showed a maximum difference after $\mathrm{d}_{\max }$ of $0.49 \%$ from the reference condition. Moreover, the minimum dose difference from the reference beam $(0.26 \%)$ was observed with the beam with a $3 \mathrm{~mm}$ radial width at the FWHM. In addition, no significant difference was observed between the PDD curves with respect to beam radial width (Figure 3).

The lateral profile showed an increased horn as the electron beam radial width at the FWHM decreased (Figure 3). Figure 3 also shows that the initial electron beam radial distribution is inversely proportional to the profile dose values. Compared with the reference beam, the average dose difference in the horn region increased from $1.49 \%$ to $15.10 \%$ as the beam radial width increased from $1 \mathrm{~mm}$ to $4 \mathrm{~mm}$ at the FWHM. The maximum and minimum differences in the horn were estimated at $23.67 \%$ and $3.28 \%$ from the reference beam when the FWHM of the beam width was $4 \mathrm{~mm}$ and 1 $\mathrm{mm}$, respectively. In addition, the overall profile dose values decreased on average by $8.42 \%$ when the radial width increased from $0 \mathrm{~mm}$ to $4 \mathrm{~mm}$ at the FWHM. The results are in agreement with those of previous studies reporting that the radial distribution of the electron beam was inversely pro- portional to beam flatness and affected the PDD less than the profile $[9,10]$.

\section{3) Energy spread of the initial electron pencil beam}

Figure 4 shows the changes in PDD and lateral dose profile as the initial electron energy spread was varied. Similar to the results described in section Results 1), the PDD values for regions deeper than $d_{\max }$ increased as the energy spread broadened. The electron beam with an energy spread of $1.019 \mathrm{MeV}$ at the FWHM showed the largest average difference from the reference beam, $2.77 \%$. The minimum difference was $0.17 \%$ and was found with an energy spread of $0.127 \mathrm{MeV}$ at the FWHM.

For the lateral profile, the curves showed dose differences averagely about $3 \%$ compared with the reference beam (FWHM of $0 \mathrm{MeV}$ ), which are comparable to the results of the other electron parameters (Figure 4). Among the energy spread conditions, the beam with an energy spread of 0.51 $\mathrm{MeV}$ at the FWHM showed the maximum average relative difference of $3.18 \%$ from the reference condition. The minimum difference of $1.48 \%$ was observed when the beam had an energy spread of $0.127 \mathrm{MeV}$ at the FWHM. The beam with the largest energy spread in this study, an FWHM of 1.019 $\mathrm{MeV}$, resulted in a difference of $2.51 \%$ on the average from 

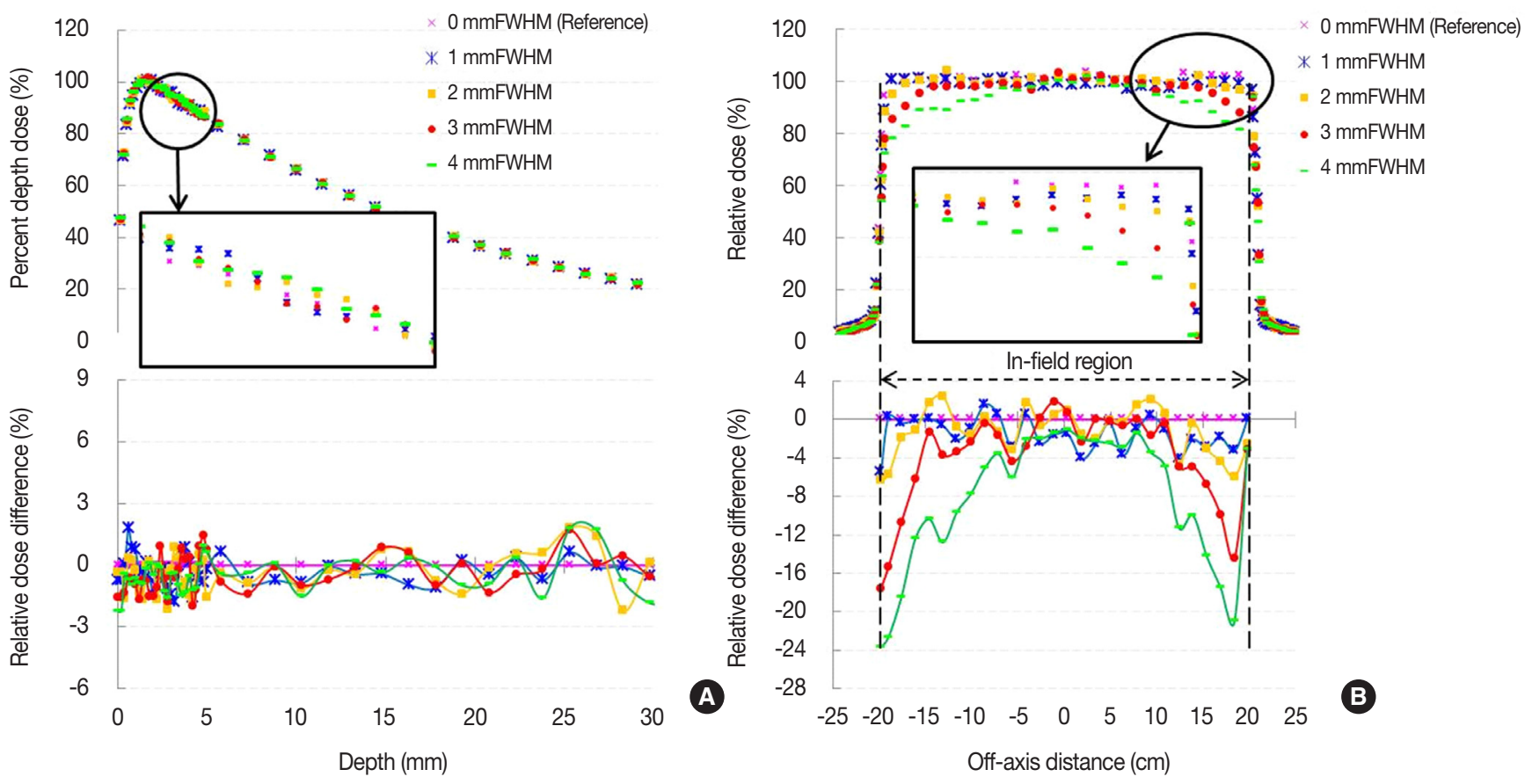

Fig. 3. Relative dose difference of percent depth dose (A) and lateral dose profile (B) by initial electron beam radial width compared to the reference initial electron beam.
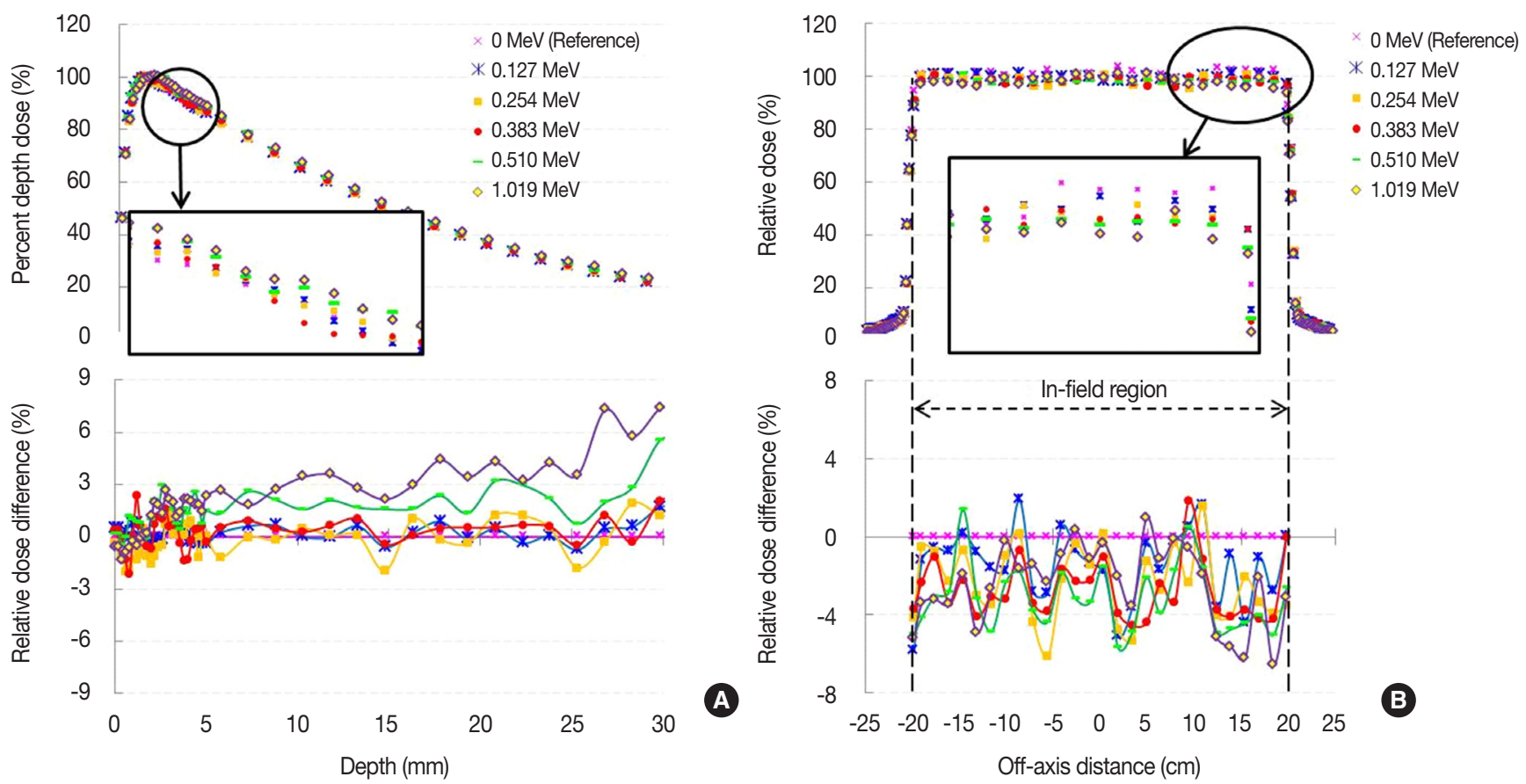

Fig. 4. Relative dose difference from the GBD for percent depth dose (A) and lateral profile (B) according to initial electron beam energy spread.

the reference condition, which corresponded to the median of the energy spreads. The differences between the energy spreads could represent statistical fluctuation in the simula- tion, considering the dose differences shown in Figure 4. These findings indicate that the energy spread cannot significantly affect the lateral dose distribution. 

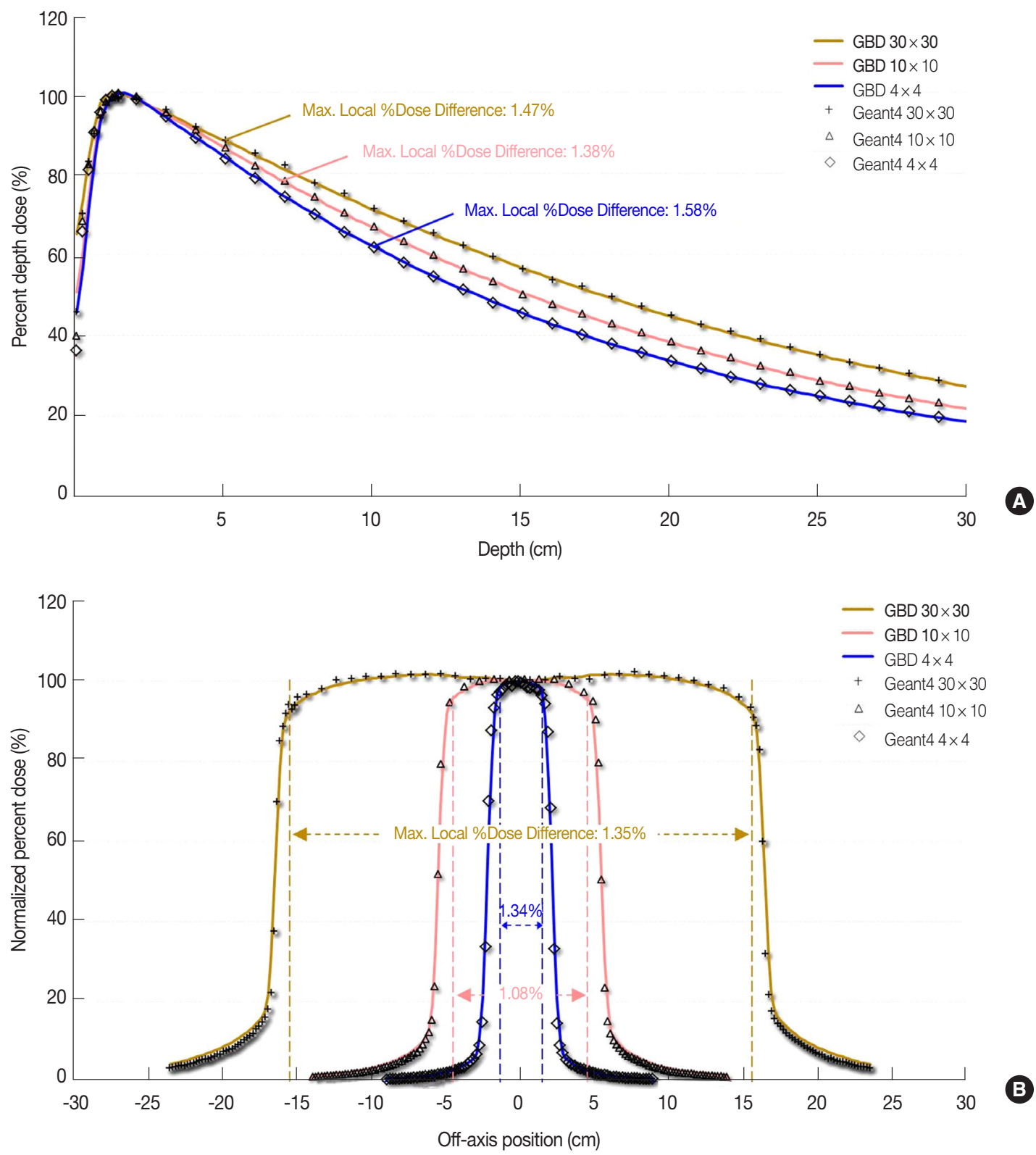

Fig. 5. Comparison of the commissioned percent depth dose (A) and lateral dose profile (B) with those of the GBD.

\section{MC commissioning of the photon beam}

Figure 5 presents the beam commissioning results carried out for field sizes of $4 \times 4 \mathrm{~cm}^{2}, 10 \times 10 \mathrm{~cm}^{2}$, and $30 \times 30 \mathrm{~cm}^{2}$. A total of $2.8 \times 10^{8}, 5.6 \times 10^{8}$ and $5.6 \times 10^{8}$ initial electrons, respectively, were generated by beams with field sizes of $4 \times 4$ $\mathrm{cm}^{2}, 10 \times 10 \mathrm{~cm}^{2}$, and $30 \times 30 \mathrm{~cm}^{2}$. The calculation of the dose distributions for the three field sizes required 39, 270, and 860 hours, respectively, with a single CPU.

The initial electron characteristics were determined by matching the PDD and the lateral profile with those of the GBD. Since the mean energy affects both the PDD and the lateral profile simultaneously, the mean electron energy was determined first, and all other parameters were determined later. The optimized initial electron parameters were 5.9 $\mathrm{MeV}, 2.5 \mathrm{~mm}$, and $0.8268 \mathrm{MeV}$ for the electron energy, beam radial width, and energy spread, respectively.

To evaluate the availability of the beams commissioned in this study, the maximum local dose difference from the GBD was calculated for each field size (Figure 5). The build-up region was not included in the PDD comparison due to its high statistical fluctuation in the MC simulation. When comparing the lateral dose profiles, the penumbra and dose fall-off 
region were not considered. The profile depth was set to 10 $\mathrm{cm}$ from the phantom surface, according to the recommendations in the AAPM Task Group No. 51 (TG-51) report [21]. Maximum local dose differences between the commissioning results and the GBD results were assessed to be less than $2 \%$ for both the PDDs and the lateral profiles.

\section{Discussion}

To accurately simulate photon beams from a LINAC head and calculate the dose distribution, it is important to determine the appropriate initial electron parameters. The quality of the initial electron beam depends on the geometric accuracy of the LINAC model. The initial electron characteristics can even differ because of minor differences in the geometry. The geometry information offered by vendors is sufficient to satisfy the required geometric similarity between the MC LINAC model and the actual LINAC machine. However, little information is available regarding the initial electron beam properties, which hinders decision-making for initial electron parameters.

Electron parameters are typically decided through empirical approaches, which are time-consuming. Since each initial electron parameter is optimized separately in the beam commissioning process, the lack of parameter knowledge complicates this process. Thus, for accurate and efficient beam commissioning, the effects of various electron parameters on beam quality should be determined. In this study, we assessed the influence of three initial electron characteristics (mean energy, energy spread, and radial intensity distribution) on PDD and lateral dose profile. We also outlined a process for electron parameter determination. Based on our results, we commissioned the initial electron beam of the Geant4-modeled LINAC.

At depths deeper than $\mathrm{d}_{\max }$, PDD increased when the mean electron energy and the FWHM of the energy spread increased. The higher mean energy of the initial electron generates higher bremsstrahlung photon energy in the target, enabling the photons to produce secondary electrons that penetrate water more efficiently and can deliver their energy to deeper locations along the beam direction. Depths shallower then $\mathrm{d}_{\max }$, which corresponded to the dose difference from the reference condition, showed larger statistical uncertainty compared to deeper locations. In the case of the energy spread, larger energy spread indicates a higher likelihood of producing electrons with both higher and lower en- ergies. Electrons with lower energies produce relatively low energy bremsstrahlung photons in the target that consists of tungsten. They showed less effect on the PDD compared to the photons generated by higher energy electrons because most of them cannot penetrate the target and reach the phantom, even though larger energy spread results in electron production with much broader energy variation than smaller energy spread. With this respect, a larger energy spread results in increased PDD values only in the region deeper than $\mathrm{d}_{\max }$. However, the dose differences from the reference condition as the energy spread was varied were smaller than those observed when the mean electron energy was varied, even though the mean energy was not varied in a wider range than that of the energy spread. Potential explanations for the findings include: (a) the energy spread follows a Gaussian distribution; (b) the majority of electrons had energy near the mean value; and (c) the mean electron energy of the spread was not changed, although the standard deviation of the energy distribution changed. The PDD was changed insignificantly according to the change of the intensity distribution of the electron beam. While changing the initial electron distribution on the target surface results in changes of the treatment beam width, it does not affect the energy of the bremsstrahlung photons produced in the target. Since the depth dose distribution is mainly affected by the bremsstrahlung photon energy, the radial intensity distribution of the initial electron beam could not have any significant effect on the PDD.

For the lateral dose profile, the lateral dose horn was inversely proportional to both the mean electron energy and the radial beam width. Compared with the reference initial electron beam, the dose values in the horn region decreased. The average dose values of the overall profiles were also decreased due to the increased mean electron energy and radial width. The increased initial electron beam energy delivered a decreased dose near the region at the edge of the field, referred to as the horn. One potential explanation for this finding is that the higher energy photons tend to be less scattered in the flattening filter and to deliver higher intensity energy in the middle of the dose profile than in the horn region. In contrast, the energy spread of the initial electron could not remarkably affect the shape of the dose profile. A broader energy spread indicates an increased initial electron energy range. As mentioned above, however, the energy spread follows a Gaussian distribution, and many of the electrons delivered into the target have an energy near the 
mean energy, even though there is an increased possibility that electrons are produced with high or low energies that are far from the center value.

Our results indicate that mean electron energy is the most important factor in determining the dose distribution; that is, the mean energy simultaneously affects the entire PDD shape and the profile. Thus, we recommend matching the overall PDD shapes and profile to the desired measurement by adjusting the mean electron energy and correcting the other details using the beam radial width and energy spread.

The optimal electron parameters determined through the MC beam commissioning process were as follows: mean energy, radial width, and energy spread; $5.9 \mathrm{MeV}, 2.5 \mathrm{~mm}$ at the FWHM, and $0.8268 \mathrm{MeV}$ at the FWHM, respectively. The dose distribution of the commissioned beam was compared with that of the GBD to demonstrate the dose availability. As shown in Figure 5, the maximum local difference from the GBD was less than $2 \%$, indicating that the beam yielded an available dose distribution on the MC simulation.

\section{Conclusion}

In this study, we investigated the relationship between the characteristics of the initial electron and the dose distribution of the LINAC beam. Our findings could be applicable for MC-based modeling of LINAC beams in the same model and also in other models. Based on these results, future studies will focus on developing more efficient beam commissioning protocols in MC simulations. In addition, the beam modeled in this study can be used for cutting-edge radiation therapy techniques using LINAC beams, such as IMRT and VMAT.

\section{Acknowledgments}

This work was supported by the National Research Foundation of Korea (NRF) grant funded by the Korea government (MSIP) (No. 2015R1C1A1A02036331).

\section{References}

1. Ma CM, et al. Monte Carlo verification of IMRT dose distributions from a commercial treatment planning optimization system. Phys. Med. Biol. 2000;45(9):2483-2495.

2. da Rosa LA, Cardoso SC, Campos LT, Alves VGL, Batista DVS, Facure A. Percentage depth dose evaluation in heterogeneous media using thermoluminescent dosimetry. J Appl. Clin. Med. Phys. 2010;11(1):117-127.

3. Sterpin E, Tomsej M, De Smedt B, Reynaert N, Vynckier S. Monte Carlo evaluation of the AAA treatment planning algorithm in a heterogeneous multilayer phantom and IMRT clinical treatments for an Elekta SL25 linear accelerator. Medical Physics. 2007;34(5):1665-1677.

4. Chetty IJ, et al. Report of the AAPM Task Group No. 105: Issues associated with clinical implementation of Monte Carlo-based photon and electron external beam treatment planning. Medical Physics. 2007;34(12):4818-4853.

5. Verhaegen F, Seuntjens J. Monte Carlo modelling of external radiotherapy photon beams. Phys. Med. Biol. 2003;48(21):R107R164.

6. Francescon P, Cora S, Chiovati P. Dose verification of an IMRT treatment planning system with the BEAM EGS4-based Monte Carlo code. Medical Physics. 2003;30(2):144-157.

7. Bush K, Gagne IM, Zavgorodni S, Ansbacher W, Beckham W. Dosimetric validation of Acuros XB with Monte Carlo methods for photon dose calculations. Medical Physics. 2011;38(4):22082221.

8. Han T, Mikell JK, Salehpour M, Mourtada F. Dosimetric comparison of Acuros XB deterministic radiation transport method with Monte Carlo and model-based convolution methods in heterogeneous media. Medical Physics. 2011;38(5):2651-2664.

9. Björk P, Knöös K, Nilsson P. Influence of initial electron beam characteristics on Monte Carlo calculated absorbed dose distributions for linear accelerator electron beams. Phys. Med. Biol. 2002;47(22):4019-4041.

10. Tzedakis A, Damilakis JE, Mazonakis M, Stratakis J, Varveris H, Gourtsoyiannis N. Influence of initial electron beam parameters on Monte Carlo calculated absorbed dose distributions for radiotherapy photon beams. Medical Physics. 2004;31(4):907-913.

11. Maskani R, Tahmasebibirgani MJ, HoseiniGhahfarokhi M, Fatahiasl J. Determination of Initial Beam Parameters of Varian 2100 CD LINAC for Various Therapeutic Electrons Using PRIMO. Asian Pac. J Cancer Prev. 2014;16(17):7795-7801.

12. Grevillot L, Frisson T, Maneval D, Zahra N, Badel JN, D Sarrut D. Simulation of a 6 MV Elekta Precise LINAC photon beam using GATE/GEANT4. Phys. Med. Biol. 2011;56(4):903-918.

13. Oliveria ACH, Vieira JW, Santana MG, Lima FR. Monte Carlo simulation of a medical linear accelerator for generation of phase spaces. 2013 International Nuclear Atlantic Conference. Recife, Brazil. November 24-29, 2013.

14. Aljarrah K, Sharp GC, Neicu T, Jiang SB. Determination of the initial beam parameters in Monte Carlo linac simulation. Medical Physics. 2006;33(4):850-858.

15. Carrier J, Archambault L, Beaulieu L, Roy R. Validation of Geant4, and object-oriented Monte Carlo toolkit, for simulations in medical physics. Medical Physics. 2004;31(3):484-492. 
16. Murakami K, et al. Systematic comparison of electromagnetic physics between Geant 4 and EGS4 with respect to protocol data. 2004 IEEE Nuclear Science Symposium Conference. Rome, Italy. October 4, 2004.

17. Keall P, Siebers JV, Libby B, Mohan R. Determining the incident electron fluence for Monte Carlo-based photon treatment planning using a standard measured data set. Medical Physics. 2003; 30(4):574-582.

18. Tanabe E, Hamm RW. Compact multi-energy electron linear accelerators. Nucl. Instrum. Methods Phys. Res., Sect. B. 1985;10: 871-876.
19. Ivanchenko V, et al. Recent improvements in Geant4 electromagnetic physics models and interfaces. Prog. Nucl. Sci. Technol. 2011;2:898-903.

20. Pandola L, Andenna C, Caccia B. Validation of the Geant4 simulation of bremsstrahlung from thick targets below $3 \mathrm{MeV}$. Nucl. Instrum. Methods Phys. Res., Sect. B. 2015;350:41-48.

21. Almond PR, Biggs PJ, Coursey BM, Hanson WF, Huq MS, Nath R, Rogers DWO. AAPM's TG-51 protocol for clinical reference dosimetry of high-energy photon and electron beams. Medical Physics. 1999;26(9):1847-1870. 